\title{
Secondary bacterial infections in patients with coronavirus disease 2019-associated pneumonia
}

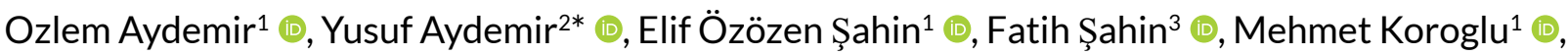 \\ Ali Fuat Erdem ${ }^{3}$ (B)
}

\begin{abstract}
SUMMARY
OBJECTIVE: The vast majority of patients who hospitalized with coronavirus disease 2019 are given empirical antibiotic therapy. However, information on the frequency, microorganism species, and resistance rates of secondary bacterial infections in coronavirus disease 2019 patients are insufficient. We aimed to show the frequency of secondary infections and resistance conditions in patients with coronavirus disease 2019 hospitalized in the intensive care unit.

METHODS: The results of tracheal aspirate culture, blood culture, and urine culture obtained from coronavirus disease 2019 patients - at least 2 days after their admission to the intensive care unit - were examined microbiologically.

RESULTS: A total of 514 patients hospitalized in intensive care unit were included in our study. Tracheal aspirate, blood, or urine cultures were collected from 369 patients (71.8\%). Bacterial reproduction was detected in at least one sample in 171 (33.3\%) of all patients. The rate of respiratory tract infection and/or bloodstream infection was found to be $21 \%$. Acinetobacter baumannii, Klebsiella pneumoniae, and Pseudomonas aeruginosa in tracheal aspirate culture; Coagulase-negative staphylococci, K. pneumoniae, and A. baumannii in blood culture; and Escherichia coli, K. pneumoniae, and Enterococcus faecalis in urine culture were the most common microorganisms. A. baumannii was resistant to most antibiotics except colistin and P. aeruginosa strains were resistant to most antibiotics except amikacin, colistin, cefepime, and imipenem. In K. pneumoniae, the highest meropenem sensitivity (73\%) was observed; there was a strong resistance to most of the remaining antibiotics.

CONCLUSIONS: We think that our study can be useful in choosing empirical antibiotic therapy in the coronavirus disease 2019 pandemic and reducing the mortality that may occur with secondary infection.

KEYWORDS: COVID-19. Secondary infection. Nasocomial infections. Antimicrobial Susceptibility.
\end{abstract}

\section{INTRODUCTION}

Among patients with coronavirus disease 2019 (COVID-19), $5-15 \%$ have moderate or severe symptoms and require hospitalization, and some require intensive care unit (ICU) follow-up. Prolonged ICU stays and use of immunosuppressor treatment regimens, such as steroids and interleukin inhibitors, may increase the frequency of secondary bacterial infections (SBIs) in COVID-19 patients $^{1}$. SBI occurs in many COVID-19 patients and is associated with worse outcomes, including death2 ${ }^{2}$.

SBIs have become the hidden threat behind COVID-193. Although most patients with COVID-19 are given empirical antibiotic therapy, there is no enough information about the type of bacterial agents that develop in these patients and their antibiotic susceptibility ${ }^{4}$. Therefore, data obtained from other viral pneumonia treatments are considered when choosing the antibiotics. During the pandemic period, different antimicrobial treatment protocols were recommended and, accordingly, many broad-spectrum antibacterial agents were used in empirical treatment before SBIs were confirmed in the majority of patients. We are using more antibiotics in our fight to save COVID-19 patients from bacterial coinfections, and it is important to consider how this could affect the prevalence of antibiotic-resistant bacteria globally. The widespread application of broad-spectrum antibacterial agents can lead to changes in antimicrobial resistance ${ }^{1,5}$. Therefore, data on the frequency and resistance characteristics of SBIs in patients hospitalized for COVID-19 are needed ${ }^{6}$. In a small number of studies on COVID-19 and SBIs, only the bacterial distribution was specified, but there are no enough data on resistance rates. Therefore, these studies are not sufficient to guide the empirical use of antibacterial agents ${ }^{3,4}$.

Hoping that the information obtained can guide empirical treatment planning and contribute to the prevention of future antimicrobial resistance, in our study, we aimed to evaluate the

\footnotetext{
${ }^{1}$ Sakarya University, Faculty of Medicine, Department of Medical Microbiology - Sakarya, Turkey.

2Sakarya University, Faculty of Medicine, Department of Pulmonology - Sakarya, Turkey.

${ }^{3}$ Sakarya University, Faculty of Medicine, Department of Anesthesiology and Reanimation - Sakarya, Turkey.

*Corresponding author: dryaydemir@yahoo.com

Conflicts of interest: the authors declare there is no conflicts of interest. Funding: none.

Received on September 19, 2021. Accepted on October 23, 2021.
} 
frequency of SBI agents and resistance conditions in patients hospitalized in ICUs with the diagnosis of COVID-19.

\section{METHODS}

This retrospective study was carried out in 514 patients who were treated for lung health and diseases and who were anesthetized in ICU in the Sakarya University Education and Research Hospital, Turkey, in March 2019 and February 2021, with a proven diagnosis of COVID-19. The study protocol was approved by the local ethics committee of the Sakarya University School of Medicine.

Demographic data of the patients, onset time of the disease and duration of hospitalization, current antibiotic treatment, and laboratory findings were analyzed retrospectively.

The results of tracheal aspirate culture, blood culture, and urine culture obtained from COVID-19 patients - at least 2 days after their admission to the ICU - were examined microbiologically. Wound and stool cultures were not included. Other sterile body fluid culture samples were not included in the evaluation when they were few in number.

All endotracheal aspirate samples were gram-stained and studied by microbiologists. If the same agent was grown in at least two sets of blood cultures taken simultaneously, it was accepted as a factor. Blood culture samples taken in a single bottle were not included in the study.

All samples taken to the laboratory were planted in sheep blood agar, chocolate agar, and eosin methylene blue agar. Plates were incubated at $35-37^{\circ} \mathrm{C}$ for $24-48 \mathrm{~h}$. Identification of the isolates grown at the end of the incubation at species level was made by MALDI-TOF/MS. Antimicrobial susceptibility studies of the isolates identified at the species level were performed using VITEK 2. Along with clinical findings, colony growth over $10^{4} \mathrm{CFU} / \mathrm{mL}$ in tracheal aspirate culture and over $10^{5} \mathrm{CFU} / \mathrm{mL}$ in urine culture was considered as infection.

Antimicrobial susceptibility results were evaluated according to EUCAST criteria.

All patients with microorganisms in culture were evaluated clinically. Samples considered as contamination/normal flora organisms and interpreted as colonizations were not accepted as SBI.

\section{RESULTS}

A total of 514 patients, 324 men and 190 women, hospitalized in ICUs with a mean age of 68.9 years, were included in our study.

All patients who had reverse transcription-polymerase chain reaction testing confirmed the diagnosis of COVID-19associated pneumonia and were transferred to ICU according to standard criteria. The average number of days of hospitalization in ICU was 8.3. Notably, 336 (65.4\%) of patients died during the 1 month follow-up; 173 of them (33.7\%) were transferred to the service by improving their general condition. Tracheal aspirate, blood, or urine cultures were obtained from 369 patients $(71.8 \%)$. Bacterial reproduction was detected in at least one sample in $171(33.3 \%)$ of all patients. Simultaneous reproduction was detected in more than one sample of 26 (5.0\%) patients. Secondary fungal infection was developed in six patients (1.1\%). There was a growth of pathogenic microorganisms in at least one of the tracheal fluid or blood cultures of 108 (21\%) patients. While $106(61.9 \%)$ patients who developed SBI resulted in death, 65 (38\%) of them were transferred to another service. The results are shown in Table 1.

Reproduction was detected in the blood culture of 72 patients who developed SBI, in the tracheal aspirate culture of 45 patients, and in the urine culture of 80 patients.

Table 1. Demographic, clinical, and microbiological characteristics of patients.

\begin{tabular}{|c|c|c|c|}
\hline Sex & & \multirow{2}{*}{$324(63) / 190(37)$} & \\
\hline \multirow[t]{2}{*}{ Men/women, n (\%) } & & & \\
\hline & Mean & Median & $\mathrm{SD}(\min / \max )$ \\
\hline Age & 68.9 & 71 & $13.9(20 / 100)$ \\
\hline Hospital day & 8.35 & 6 & $8.9(1 / 89)$ \\
\hline \multirow{3}{*}{ Survival status, n (\%) } & Exitus & Transferred to non-ICU & Already stayed in ICU \\
\hline & $336(65.4)$ & $173(33.7)$ & $5(1)$ \\
\hline & Present & Absent & Not taken as an example \\
\hline Reproduction in any sample & 171(33.3) & $198(38.5)$ & $145(28.2)$ \\
\hline Reproduction in tracheal aspirate & $45(8.75)$ & $27(5.25)$ & $442(86)$ \\
\hline Reproduction in blood culture & $72(14)$ & $199(38.7)$ & $243(47.3)$ \\
\hline Reproduction in urine culture & $80(15.6)$ & $147(28.6)$ & $287(55.8)$ \\
\hline
\end{tabular}


The tracheal aspirate culture was obtained from 72 (14\%) patients according to the criteria of high fever, C-reactive protein, procalcitonin, white blood cell increase, consolidation areas on lung X-ray, and increased secretion amount. There was no reproduction in seven $(9.7 \%)$ of them. Upper respiratory tract flora elements were produced in 20 (27.8\%) of them. Acinetobacter baumannii was the most common microorganism grown in tracheal aspirate culture in $13(18 \%)$ patients with COVID19. This was followed by Klebsiella pneumoniae in nine (12.5\%) patients and by Pseudomonas aeruginosa in five (6.9\%) patients. Multiple microorganisms were detected in five patients.

Blood culture was obtained from 271 (52.8\%) of patients. Reproduction was not detected in 199 (73.4\%) patients, while reproduction was observed in $72(26.5 \%)$ of them. When the factors grown in blood culture were evaluated, Staphylococcus epidermidis was grown in 19 (7\%) patients. The second most common was $K$. pneumoniae in 15 (5.5\%) patients. Subsequently, $A$. baumannii was detected in six (2.2\%) patients and Enterococcus faecalis in five $(1.8 \%)$ patients.

Urine culture was obtained from 227 (44.1\%) patients. Reproduction was found in 80 (35.7\%) of them. Escherichia coli was isolated in $21(9.3 \%)$ and K. pneumoniae in 21 (9.3\%) of the urine samples with reproduction. E. faecalis was detected in $11(4.8 \%)$ of the samples.

The distribution of microorganisms is shown in Table 2.

When all sample regions were included, $K$. pneumoniae (26.3\%) was the most frequently isolated agent from all samples, followed by E. coli (15.7\%), A. baumannii (12.9\%), and S. epidermidis (11.1\%). Candida reproduction was observed in only six of patients (Table 2).

When the antimicrobial resistance of the bacteria grown was evaluated, all $A$. baumannii strains were found to be resistant to meropenem, imipenem, ciprofloxacin, levofloxacin, piperacillin, tazobactam, ceftazidime, and cefepime, while $68 \%$ of the strains were resistant to amikacin, $68 \%$ to trimethoprim sulfamethoxazole, and $88 \%$ to gentamicin. All strains were found to be susceptible only to colistin.

In $P$. aeruginosa, all strains were found to be susceptible to amikacin, whereas colistin resistance was detected in only one strain. Also, 64.2\% (nine strains) of strains were found to be resistant to piperacillin and tazobactam, $35.7 \%$ (five strains) to gentamicin, and $28.5 \%$ (four strains) to imipenem.

In $K$. pneumoniae, $6.72 \%$ of strains were resistant to imipenem, $62.2 \%$ to ceftazidime, and $66.7 \%$ to ciprofloxacin.

The results of antimicrobial susceptibility of $A$. baumannii, $P$. aeruginosa, $K$. pneumoniae, and $E$. coli strains are shown in Table 3.

Methicillin resistance was observed in 64\% of Staphylococcus strains. All strains were susceptible to vancomycin and teicoplanin.

\section{DISCUSSION}

It is a long-known fact that viral infections increase the frequency of SBIs and the morbidity and mortality rates. For example, in the $2009 \mathrm{H} 1 \mathrm{~N} 1$ influenza pandemic, bacterial infection secondary to viral infection was developed in $20-30 \%$ of patients, which increased mechanical ventilation and mortality rates ${ }^{7}$. In the light of these experiences from past viral outbreaks, empirical antibiotic treatment is initiated in more than $70 \%$ of hospitalized COVID-19 patients ${ }^{4}$. However, studies showing the frequency of SBIs in COVID-19, the diversity of microorganisms, and their resistance status are very few ${ }^{2,5,6}$. To know the frequency and resistance rates of secondary infections that significantly increase mortality, especially during pandemic periods, antibiotic selection is of great importance in terms of patient management, correct use of resources, and prevention

Table 2. Types of microorganisms that grow according to sample location.

\begin{tabular}{|c|c|c|c|c|}
\hline$n(\%)$ & Tracheal aspirate & Blood & Urine & Total (n/\%) \\
\hline Acinetobacter baumannii & 13 & 6 & 5 & 22/12.9 \\
\hline Klebsiella pneumoniae & 9 & 15 & 21 & $45 / 26.3$ \\
\hline Pseudomonas aeruginosa & 5 & 2 & 2 & 9/5.3 \\
\hline Staphylococcus aureus & 4 & 4 & 1 & $9 / 5.3$ \\
\hline Staphylococcus epidermidis & - & 19 & 1 & 20/11.7 \\
\hline Enterococcus faecalis & - & 5 & 11 & $16 / 9.4$ \\
\hline Escherichia coli & 1 & 1 & 27 & 29/15.7 \\
\hline Corynobacterium spp & 3 & 5 & - & $8 / 4.7$ \\
\hline Candida albicans & - & 2 & 4 & $6 / 3.5$ \\
\hline Staphylococcus hominis & - & 4 & & $4 / 2.4$ \\
\hline Pseudomonas aeruginosa+Enterococcus faecalis & 2 & - & 1 & $3 / 1.8$ \\
\hline
\end{tabular}


Table 3. Antimicrobial susceptibility results of microorganisms.

\begin{tabular}{|c|c|c|c|c|}
\hline & $\begin{array}{c}\text { Acinetobacter baumannii } \\
\text { n: } 28(\%)\end{array}$ & $\begin{array}{c}\text { Pseudomonas aeruginosa } \\
\text { n: } 14(\%)\end{array}$ & $\begin{array}{l}\text { Klebsiella pneumoniae } \mathrm{n} \text { : } \\
45(\%)\end{array}$ & $\begin{array}{c}\text { Escherichia coli } \\
\text { n: } 21(\%)\end{array}$ \\
\hline Colistin & $28(100)$ & 13 (92.9) & - & - \\
\hline Tmp/smx & 9 (32.1) & - & 24 (53.3) & $14(66.7)$ \\
\hline Amikacin & $8(28.6)$ & $14(100)$ & $21(46.7)$ & $19(90.5)$ \\
\hline Gentamycin & $3(10.7)$ & $9(64.3)$ & $27(60)$ & $17(81)$ \\
\hline Tigecycline & 3 (10.7) & - & - & - \\
\hline Cefepim & - & 11 (78.6) & 24 (53.3) & $12(57.1)$ \\
\hline Imipenem & 9 (32.1) & $10(71.4)$ & 24 (53.3) & $21(100)$ \\
\hline Ceftazidime & $3(10.7)$ & 9 (64.3) & $19(42.2)$ & $14(66.7)$ \\
\hline Meropenem & 9 (32.1) & 8 (57.1) & 33 (73.3) & 33 (73.3) \\
\hline Ciprofloxacin & 8 (28.6) & 8 (57.1) & 23 (51.1) & 8 (38.1) \\
\hline Levofloxacin & 8 (28.6) & $7(50)$ & $22(48.9)$ & 13 (61.9) \\
\hline Ertapenem & 9 (32.1) & - & 31 (68.9) & 31 (68.9) \\
\hline
\end{tabular}

of resistance development ${ }^{6,8}$. In the post-COVID era, increased global antibiotic resistance could be a potential public health problem 9 . Therefore, collecting clinical data on secondary infections and antibiotic resistance is important and almost critical ${ }^{1,7}$.

In our study, we investigated the frequency of SBIs and resistance status in patients hospitalized in ICUs with the diagnosis of COVID-19.

The rate of secondary respiratory tract and/or bloodstream infection was found to be $21 \%$ in our study. Our results are coherent with reports from available cohorts, where the proportion of patients with secondary infections ranged from 5 to $30 \% \%^{3,4,10,11}$. In our study, the urinary system infection rate was found to be $15.6 \%$, bloodstream infection rate was $14 \%$, and respiratory system infection rate was $8.4 \%$. A. baumannii, K. pneumoniae, and $P$. aeruginosa in tracheal aspirate culture; coagulase-negative staphylococus, $K$. pneumoniae, and A. baumannii in blood culture; and E. coli, K. pneumoniae, and E. faecalis in urine culture were the most common microorganisms. When we observed the resistance rates, $A$. baumannii was resistant to most antibiotics except colistin. P. aeruginosa strains were resistant to most antibiotics except amikacin, colistin, cefepim, and imipenem. In K. pneumoniae, the highest meropenem sensitivity (73\%) was observed, and there was a resistance to most of the remaining antibiotics.

The rates of resistant bacteria in ICUs vary between countries and hospitals. The results of our study were similar to the period before COVID-19, except for the high rate of $A$. baumannii. The reason for the high rates of Acinetobacter can be explained by the longer hospitalization period and more steroid and immunosuppressive use in COVID-19 patients.
In early studies, SBI was reported in 5-27\% of all COVID19 patients and $13.5-44 \%$ of those hospitalized patients in the $\mathrm{ICU}^{3,12,13}$. Among the pathogens responsible for the development of these infections, it is observed that multidrug resistant bacteria are common ${ }^{1,4}$. Among the microorganisms isolated from patients, A. baumannii, carbapenem-resistant enterobacteriaceae, K. pneumoniae, P. aeruginosa, Candida albicans, and Candida glabrata that produce broad spectrum $\beta$-lactamase (ESBL) are the most frequently detected microorganisms $s^{13,14}$. However, according to earlier studies, changes can be observed in the frequency of detected microorganisms. For example, in the Zhou et al. ${ }^{9}{ }^{6}$ study, SBI was detected in 28 (14\%) of 191 patients, and 27 of them died. Coexistence of SARS-CoV-2 and bacterial infection increases mortality at a high rate ${ }^{1,2,3}$. In our center, $62 \%$ of patients who developed SBI resulted in death.

Currently available clinical data show that patients infected with SARS-CoV-2 have a lower bacterial or fungal co-infection rate than other viral infections. The reason for this may be that the healthcare personnel providing care and treatment services are highly adapted to the use of personal protective equipment and hand disinfection, because secondary infections may develop due to microorganisms carried by medical equipment or personnel, especially in patients hospitalized in ICUs. Another reason may be the use of empirical antibiotics in the early period of SARS-CoV-2 infection.

Viral infections damage the respiratory tract both histologically and functionally ${ }^{1}$. Damage to ciliary cells can lead to impaired mucociliary clearance, increased adhesion of bacteria to mucins, and increased colonization of bacteria in the 
airway $^{15}$. Increased susceptibility to secondary infections may vary depending on the severity of the disease, the type of virus, and the bacterial strain. However, the prevalence and microbiology of concomitant bacterial infections in patients with SARS$\mathrm{CoV}-2$ infection are not yet fully understood. The number of studies on this subject is relatively small ${ }^{1,2,5,6}$.

The strength of our study is that it provides comprehensive data on this subject because we do not have sufficient information, including antibiotic resistance status. Our study has some limitations. The first of these limitations is that invasive procedures such as central venous or arterial catheters cannot be clearly defined and distinguished. Since the majority of patients were critically ill, they had a catheter and were mechanically ventilated. Second, since we are a pandemic center, we could not establish a control group with nonCOVID-19 patients from the same period. Therefore, we could not certainly state the percentages of bacterial growth in our patients caused by COVID-19.

\section{REFERENCES}

1. Bengoechea JA, Bamford CG. SARS-CoV-2, bacterial co-infections, and AMR: the deadly trio in COVID-19? EMBO Mol Med. 2020;12(7):e12560. https://doi.org/10.15252/emmm.202012560

2. Silva DL, Lima CM, Magalhães VCR, Baltazar LM, Peres NTA, Caligiorne RB, et al. Fungal and bacterial coinfections increase mortality of severely ill COVID-19 patients. J Hosp Infect. 2021;113:145-54. https://doi.org/10.1016/j.jhin.2021.04.001

3. CoxMJ, Loman N, Bogaert D, O'Grady J. Co-infections: potentially lethal and unexplored in COVID-19. Lancet Microbe. 2020;1(1):e11. https://doi.org/10.1016/S2666-5247(20)30009-4

4. Langford BJ, So M, Raybardhan S, Leung V, Westwood D, MacFadden $\mathrm{DR}$, et al. Bacterial co-infection and secondary infection in patients with COVID-19: a living rapid review and meta-analysis. Clin Microbiol Infect. 2020;26(12):1622-29. https://doi.org/10.1016/j. cmi.2020.07.016

5. Mirzaei R, Goodarzi P, Asadi M, Soltani A, Aljanabi HAA, Jeda AS, et al. Bacterial co-infections with SARS-CoV-2. IUBMB Life. 2020;72(10):2097-111. https://doi.org/10.1002/iub.2356

6. Zhou P, Liu Z, Chen Y, Xiao Y, Huang X, Fan XG. Bacterial and fungal infections in COVID-19 patients: a matter of concern. Infect Control Hosp Epidemiol. 2020;41(9):1124-5. https://doi. org/10.1017/ice.2020.156

7. Chertow DS, Memoli MJ. Bacterial coinfection in influenza: a grand rounds review. JAMA. 2013;309(3):275-82. https://doi. org/10.1001/jama.2012.194139

8. Lynch C, Mahida N, Gray J. Antimicrobial stewardship: a COVID casualty? J Hosp Infect. 2020;106(3):401-3. https://doi. org/10.1016/j.jhin.2020.10.002

\section{CONCLUSIONS}

Potential management interventions should be urgently considered to support reduced antimicrobial prescribing during the COVID-19 pandemic. There are no guidelines for which antibiotic to choose in which clinical situations. Knowing the regional and global causative microorganisms and resistance characteristics will increase the success of the pandemic struggle and reduce the common resistance problem in the post-COVID period by contributing to the selection of appropriate antibiotics?

As a result, we think that our study can be useful in choosing empirical antibiotics in the COVID-19 pandemic and in reducing the mortality that may occur with secondary infection.

\section{AUTHORS' CONTRIBUTIONS}

ÖA, YA: Writing - original draft \& editing, Investigation, Software, Formal Analysis, Data curation, Visualization. EÖŞ, FŞ, MK, AFE: Investigation. ÖA: Methodology.

9. Cantón R, Gijón D, Ruiz-Garbajosa P. Antimicrobial resistance in ICUs: an update in the light of the COVID-19 pandemic. Curr Opin Crit Care. 2020;26(5):433-41. https://doi.org/10.1097/ MCC.0000000000000755

10. Ripa M, Galli L, Poli A, Oltolini C, Spagnuolo V, Mastrangelo A et al. Secondary infections in patients hospitalized with COVID19: incidence and predictive factors. Clin Microbiol Infect. 2021;27(3):451-7. https://doi.org/10.1016/j.cmi.2020.10.021

11. Garcia-Vidal C, Sanjuan G, Moreno-García E, Puerta-Alcalde P, Garcia-Pouton N, Chumbita M, et al. Incidence of co-infections and superinfections in hospitalized patients with COVID-19: a retrospective cohort study. Clin Microbiol Infect. 2021;27(1):83-8. https://doi.org/10.1016/j.cmi.2020.07.041

12. Huang C, Wang Y, Li X, Ren L, Zhao J, Hu Y, et al. Clinical features of patients infected with 2019 novel coronavirus in Wuhan, China. Lancet. 2020:395(10223):497-506. https://doi.org/10.1016/ S0140-6736(20)30183-5

13. Chen N, Zhou M, Dong X, Qu J, Gong F, Han Y, et al. Epidemiological and clinical characteristics of 99 cases of 2019 novel coronavirus pneumonia in Wuhan, China: a descriptive study. Lancet. 2020;395(10223):507-513. https://doi.org/10.1016/S01406736(20)30211-7

14. Clancy CJ, Nguyen MH. Coronavirus disease 2019 , superinfections, and antimicrobial development: what can we expect? Clin Infect Dis. 2020;71(10):2736-43. https://doi. org/10.1093/cid/ciaa524

15. Morris DE, Cleary DW, Clarke SC. Secondary bacterial infections associated with influenza pandemics. Front Microbiol. 2017;8:1041. https://doi.org/10.3389/fmicb.2017.01041 